\title{
Importance of pursuing a second opinion before arriving at final diagnosis
}

Sir,

Oral pathology is a recognized specialty of dentistry, deals with the nature, identification and management of diseases affecting the oral and maxillofacial regions. It is always a fascinating and exquisite subject in diagnosing and managing the diseases affecting the oral cavity. Oral and maxillofacial pathologists (OMP) complete a 3 year accredited training program that provides education in the microscopic and clinical diagnosis of diseases within the oral cavity and maxillofacial complex.

Medical error is a common problem and its human cost in terms of disability, suffering, and death is stunning. Steps toward reducing medical error will require the identification of mistake-prone practices within a complex health care system. Erroneous pathologic diagnosis has been identified as one source of error. ${ }^{[1]}$

Among disease sites, the head and neck can be a particularly problematic area for pathologists, prompting a frequent secondopinion, which is intended to expose clinically significant errors that have a direct impact on patient care..$^{[2]}$

A second-opinion is the process of seeking an evaluation by another doctor or surgeon to confirm the diagnosis and treatment plan of a primary physician, or to offer an alternative diagnosis and/or treatment approach..$^{[2]}$

A second review of histopathologic diagnoses is a quality assurance practice that helps expose diagnostic errors and guide management of patients. ${ }^{[3]}$ It also plays an important role in the practice of diagnostic pathology. Mandatory second-opinion pathology consistently uncovers discrepancies across all major organ systems and has a profound impact on management and prognosis. Several studies have documented the cost effectiveness and positive impact of seeking second-opinion on patient management. ${ }^{[2]}$

Site-specific studies have implicated the head and neck as a highrisk area that is prone to diagnostic error. Diagnostic discrepancy rates have ranged from $1 \%$ to $53 \%$ for surgical pathology studies and from $17 \%$ to $60 \%$ for cytopathology studies. Major changes (affecting treatment or prognosis) occur in $5-7 \%$ of surgical pathology cases. ${ }^{[1]}$

A study was done to assess the impact of second-opinion by retrieving the patient reports from 1990 to 2000 the results of which showed that out of 814 cases reviewed, the second-opinion surgical pathology diagnosis resulted in $54(7 \%)$ changed diagnoses. Of the changed diagnosis, $13(24 \%)$ involved a change from a benign to a malignant diagnosis; $8(15 \%)$ involved a change from a malignant to a benign diagnosis, and $33(61 \%)$ involved a change in tumor classification. Follow-up information supported the second-opinion diagnosis in 41 of 43 cases (95\%) that concluded that second-opinion surgical pathology results in major therapeutic and prognostic modifications for patients sent to large referral hospitals for head and neck oncologic surgery. ${ }^{[3]}$

Another study was done to determine the patterns of second-opinion requests made by physician pathologists to OMP by retrospectively reviewing submitted reports with the second diagnosis reports and this study supports the positive impact of second-opinion surgical pathology for lesions in the maxillofacial complex and supports the role of OMP in subspecialty diagnostic pathology. ${ }^{[2]}$

Another study reviewed the impact of mandatory second-opinion surgical pathology. Cases were collected prospectively over a 21 month period from April 1995 to December 1996. The majority of cases involved a change between benign and malignant or a major change in tumor classification and concluded that second-opinion surgical pathology can result in major therapeutic and prognostic modifications for patients sent to large referral hospitals. Although the overall percentage of affected cases is not large, the consistent rate of the discrepant diagnosis uncovered by second-opinion surgical pathology may have an enormous human and financial impact. ${ }^{[4]}$

The pathologist should feel free to ask for the second-opinion in case of misperception without any hesitations in mind in order to reduce the error that may affect the management.

Clinicians also should understand the problems posed by pathologist like biopsy procedures and processing of the tissues that affect the diagnosis to some extent. The clinicians and the pathologists should go hand in hand for the betterment of the patient's health. Their personnel opinion regarding each other should not affect the patient's care.

Second-opinions that agree with the first provider's conclusions may help ease the patient's mind and provide a clearer picture of the necessary course of treatment or surgery. However, if a patient still feels uncomfortable with the treatment plan outlined by the first and second-opinions, or strongly disagrees with their conclusions, a third opinion from another provider is an option.

In cases in which the second provider disagrees with the first provider on diagnosis and/or treatment, the patient has harder 
choices to face. Again, a third evaluation may be in order from yet another physician.

Also, various studies have supported the positive impact of second-opinion surgical pathology for lesions in the maxillofacial complex and supports the role of OMP in subspecialty diagnostic pathology.

In all cases, a patient should remember that their personal preferences, beliefs, and lifestyle considerations must also be considered in their final decision on surgery or treatment, as they are the ones who will live with the results.

\section{Neethu Telagi, Bangalore Rahim Ahmed Mujib}

Department of Oral Pathology and Microbiology, Bapuji Dental College and Hospital, Davanagere, Karnataka, India

Address for Correspondence:

Dr. Neethu Telagi,

Department of Oral Pathology and Microbiology, Bapuji Dental College and Hospital, Davanagere - 577 004, Karnataka, India. E-mail: neethutelagi@gmail.com

\section{REFERENCES}

1. Kronz JD, Westra WH. The role of second opinion pathology in the management of lesions of the head and neck. Curr Opin Otolaryngol Head Neck Surg 2005;13:81-4.

2. Jones K, Jordan RC. Patterns of second-opinion diagnosis in oral and maxillofacial pathology. Oral Surg Oral Med Oral Pathol Oral Radiol Endod 2010;109:865-9.

3. Westra WH, Kronz JD, Eisele DW. The impact of second opinion surgical pathology on the practice of head and neck surgery: A decade experience at a large referral hospital. Head Neck 2002;24:684-93.

4. Kronz JD, Westra WH, Epstein JI. Mandatory second opinion surgical pathology at a large referral hospital. Cancer 1999;86:2426-35.

\begin{tabular}{|l|l|}
\hline Access this article online & Website: \\
\hline Quick Response Code: & www.ijmedph.org \\
\cline { 2 - 3 } & DOl: \\
\hline & $10.4103 / 2230-8598.144140$ \\
\hline & \\
\hline
\end{tabular}

Announcement

\section{Android App}

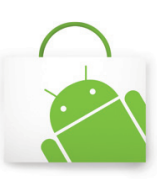

A free application to browse and search the journal's content is now available for Android based mobiles and devices. The application provides "Table of Contents" of the latest issues, which are stored on the device for future offline browsing. Internet connection is required to access the back issues and search facility. The application is compatible with all the versions of Android. The application can be downloaded from https://market.android.com/details?id=comm.app.medknow. For suggestions and comments do write back to us. 